\title{
QUALIDADE DE SOFTWARE AGROPECUÁRIO: UM ESTUDO DE CASO
}

\author{
Quality of agricultural software: a case study
}

\author{
Henri Cócaro', Marcos Aurélio Lopes², Fernanda Cláudia Alves Campos ${ }^{3}$
}

\section{RESUMO}

A qualidade de um software agropecuário é fundamental para conduzir o usuário a extrair os benefícios esperados com a sua utilização. Fez-se um estudo de caso para a avaliação de características e sub-características de qualidade de um produto de software comercial destinado ao gerenciamento de rebanhos bovinos. Concluiu-se que o software foi considerado aceitável.

Termos para indexação: Agronegócio, informática, sistemas de informação, qualidade de software, softwares agropecuários.

\begin{abstract}
The quality of an agropecuary software is essential to conduct the user to extract the expected advantages of its use. A case study for the evaluation of caracteristics and sub-caracteristics of the quality of the commercial software product for the management of beef cattles was performed. It was conclude that the software is considered acceptable.
\end{abstract}

Index terms: Agribusiness, agricultural softwares, computer science, information systems, quality of software.

\section{(Recebido para publicação em 3 de agosto de 2004 e aprovado em 10 de junho de 2005)}

\section{INTRODUÇÃO}

Qualidade é um termo que pode ser definido de várias formas, pois, não tem um sentido único. Para cada conceito existem vários níveis de abstração e a visão popular normalmente é diferente do seu uso profissional. Na visão popular, o termo é indefinível e a qualidade pode ser sentida, discutida, julgada, mas não pode ser medida. Normalmente é relacionada a luxo, classe e elegância e os produtos caros e complexos têm melhor nível de qualidade. Aspectos como confiabilidade e o número de reparos efetuados no produto não são considerados (BOAVENTURA, 2001). Do ponto de vista técnico, a Norma ISO/CD8402 define que qualidade é a totalidade das características de um produto ou serviço que lhe confere a capacidade de satisfazer as necessidades implícitas e explícitas dos usuários (ISO/IEC, 2001).

De acordo com Campos (2001), a qualidade não basta existir, ela deve ser reconhecida pelo cliente. Para facilitar esse reconhecimento existe a certificação de qualidade que é oficialmente emitida com base em padrões e está muitas vezes representada por selos de certificação de empresas autorizadas pela International Organization for Standardization - ISO por itens indicativos de conformidade com normas internacionais (ISO 9001, ISO 9002, ISO14000, QS 9000) para empresas de diferentes segmentos: implementos agrícolas, software, têxtil, segurança e outros.
A qualidade de produtos de software é tratada, entre outras, na série de Normas ISO/IEC 9126, na série ISO/IEC 14598 e na Norma ISO/IEC 12119, que descrevem requisitos de qualidade e trazem diretrizes para o processo avaliativo. A avaliação é uma atividade técnica, que se refere à ação de aplicar critérios de avaliação especificamente documentados para um módulo de software, pacote ou produto, com o propósito de determinar sua aceitação, liberação ou rejeição e contribuir para sua melhoria futura. A versão da ISO/IEC 9126 (ISO/IEC, 2001) está dividida em quatro partes: modelo de qualidade, métricas externas, métricas internas e métricas da qualidade em uso. As características externas oferecem uma visão dinâmica do software, as características internas resultam em uma visão estática do sistema e a avaliação das características da qualidade em uso ocorre quando o software é utilizado pelo usuário, para realização de suas tarefas no seu ambiente de trabalho.

O tema qualidade de software agropecuário é importante, posto que, muitas softhouses, que são as empresas desenvolvedoras de pacotes de softwares, elaboram produtos voltados para o mercado agropecuário. De acordo com Lopes (2000), houve um aumento de 54\% na oferta de softwares agropecuários em 1997, 17\% em 1999 (LOPES, 2000) e 24\% em 2004. A maioria dessas softhouses não se preocupa com a qualidade e nem mesmo procuram saber as características fundamentais que seus produtos deveriam atender. Em relação a esse mercado,

\footnotetext{
${ }^{1}$ Zootecnista, Mestrando em Administração/UFLA - Av. Evaristo Gomes Guerra 1286 / 303 - Lavras, MG - 37200-000 - henricocaro@hotmail.com. ${ }^{2}$ Professor DSc. do Departamento de Medicina Veterinária da Universidade Federal de Lavras/UFLA - Caixa postal 3037 - Lavras, MG -37200-000 malopes@ufla.br.

${ }^{3}$ Professora do Departamento de Ciência da Computação da Universidade Federal de Juiz de Fora/UFJF.
} 
especificamente de softwares para bovinos, observou-se uma retração do ano de 1997 para 1999 na quantidade de softwares. Das explicações pode-se citar duas. A primeira refere-se a baixa qualidade dos produtos, que além de não trazerem características que atendam às necessidades dos usuários, na maioria das vezes, usavam tecnologias ultrapassadas para um mercado que já apontava para sistemas centrados na Web e muitas vezes com escassez de informação sobre o agronegócio. A segunda citada por Cócaro (2004), refere-se a carência de treinamentos aos usuários oferecidos pelas softhouses, o que acabou conduzindo à baixa eficiência de uso in loco, ou seja, ao baixo uso do software dentro da empresa rural, levando a saída de empresas do ramo e como conseqüência a saída de seus produtos do mercado.

Para testar a viabilidade de uma avaliação técnica, na visão de especialistas do domínio e analisar a aplicação em produtos de software comerciais da área agropecuária, foi especificado um conjunto de atributos de qualidade e realizado um estudo de caso com um produto de software bem difundido no mercado agropecuário.

\section{MATERIAL E MÉTODOS}

Para este estudo de caso sobre avaliação da qualidade foi escolhido, aleatoriamente, um software específico para monitoramento e gerenciamento de rebanhos, aqui denominado de software ALFA, tendo como um dos critérios de seleção a disponibilidade para download na Internet, realizado em junho de 2004, e contatos com a softhouse. O software foi instalado segundo as instruções nele constadas e realizadas várias simulações de uso para explorações das suas rotinas, além de ter sido realizado contato por $e$-mail com a softhouse para saber sobre suas condições de comercialização e suporte do produto.

A metodologia usada para avaliação da qualidade, foi proposta por Campos (1999, 2001) e Campos et al. (2000) que tem pesquisado e trabalhado com um conjunto de características e sub-características aplicáveis ao amplo espectro de produtos da área, na visão do usuário. Privilegiar essa visão na avaliação dos produtos disponíveis comercialmente permite que os resultados auxiliem os usuários na seleção de produtos de qualidade e as softhouses têm a chance de melhorar seu processo de desenvolvimento e suas práticas de engenharia para atingir tais expectativas.

A metodologia apresentada nesse trabalho, que por questões de praticidade e aplicabilidade, está resumidamente descrita na forma de uma ficha de avaliação, é aderente à Norma ISO 9126, tanto no modelo de avaliação, quanto na seleção dos atributos de qualidade a serem considerados. Privilegiar a visão do usuário foi uma abordagem selecionada que direcionou a escolha das características e sub-características e seu posterior agrupamento. Dessa forma, nesse conjunto selecionado estão priorizadas as características de funcionalidade, confiabilidade, usabilidade, eficiência e manutenibilidade, aqui relacionadas em atributos inerentes ao domínio do software a ser avaliado, no caso o agropecuário. $\mathrm{O}$ fato das características de qualidade da Norma ISO 9126 não permitirem uma medição direta, havendo a necessidade da definição de métricas, exigiu dessa proposta a seleção de um conjunto de características, suas sub-características e as métricas a serem usadas no processo avaliativo (Quadro 1) e a adoção de uma escala para medir o grau de presença dessas métricas no produto a ser avaliado (Quadro 2).

O método consistiu na definição de como o atributo vai ser medido (definição das métricas), se é medida direta, usa-se sim ou não; se é medida indireta ele é avaliado por uma escala que vai de 0 a 4 . Neste trabalho consideraramse todas as características e sub-características como medidas indiretas, determinando-se uma nota (Quadro 1) para o atributo de acordo com a percepção dos dois avaliadores, especialistas do domínio, seguido da discussão para justificativa da avaliação.

\section{RESULTADOS E DISCUSSÕES}

Os resultados da avaliação do software ALFA (Quadro 3) foram estruturados na seqüência das métricas referentes a cada característica seguidos da discussão. Foi atribuído o conceito sim ou não para indicar a presença ou ausência do atributo e a nota atribuída pelos avaliadores de acordo com a escala proposta no Quadro 2.

\section{CARACTERÍSTICA - FACILIDADE DE USO}

\section{Sub-característica - Facilidade de personalização}

A interface com o usuário é facilmente personalizada para o uso por usuários de diferentes classes e tipos?

Sim, nota 4. Identificou-se uma rotina, na qual o usuário criava os parâmetros que iria trabalhar e permitia a ele escolher quais comandos, na forma de botões, desejava que fossem exibidos na tela principal. Também oferecia um nível de senha que restringia acesso à operações na parte financeira.

Ciênc. agrotec., Lavras, v. 29, n. 5, p. 1075-1082, set./out., 2005 
QUADRO 1 - Características e sub-características para avaliação da qualidade de software agropecuário - Modelo de ficha de avaliação.

\begin{tabular}{|c|c|c|c|}
\hline CARACTERÍSTICAS & SUB-CARACTERÍSTICAS & PERGUNTAS & NOTA \\
\hline \multirow[t]{2}{*}{ Facilidade de Uso } & Facilidade de personalização & $\begin{array}{l}\text { A interface com o usuário é facilmente } \\
\text { personalizada para o uso por usuários } \\
\text { de diferentes classes e tipos? }\end{array}$ & \\
\hline & Manual do usuário & $\begin{array}{l}\text { A documentação sobre o uso do } \\
\text { software é de fácil compreensão pelo } \\
\text { usuário? }\end{array}$ & \\
\hline \multirow{4}{*}{ Facilidade de operação } & $\begin{array}{l}\text { Simplicidade para registrar } \\
\text { informações }\end{array}$ & $\begin{array}{l}\text { É simples a entrada de dados de } \\
\text { natureza física, zootécnica, financeira e } \\
\text { econômica no software? }\end{array}$ & \\
\hline & $\begin{array}{l}\text { Facilidade de compreensão dos } \\
\text { resultados }\end{array}$ & $\begin{array}{l}\text { É fácil ao usuário compreender os } \\
\text { resultados parciais e finais fornecidos } \\
\text { pelo software? }\end{array}$ & \\
\hline & Facilidade de consulta dos dados & $\begin{array}{l}\text { É fácil consultar os dados } \\
\text { armazenados? }\end{array}$ & \\
\hline & Facilidade de alteração dos dados & É fácil alterar os dados? & \\
\hline \multirow{3}{*}{$\begin{array}{l}\text { Serviços oferecidos pelo } \\
\text { vendedor }\end{array}$} & Direito a upgrade & $\begin{array}{l}\text { A aquisição do software garante o } \\
\text { direito a upgrade gratuito ou a preço } \\
\text { compatível? }\end{array}$ & \\
\hline & Suporte técnico & A empresa oferece suporte técnico? & \\
\hline & Manutenção & $\begin{array}{l}\text { A empresa oferece serviço de } \\
\text { manutenção do software? }\end{array}$ & \\
\hline Integridade do sistema & Robustez & $\begin{array}{l}\text { O software é capaz de manter o } \\
\text { processamento, a despeito da } \\
\text { ocorrência de ações inesperadas } \\
\text { (entradas de dados incorretos, } \\
\text { execuções de ações indesejadas, } \\
\text { operações de efeito grave)? } \\
\end{array}$ & \\
\hline $\begin{array}{l}\text { Necessidade de adaptação do } \\
\text { sistema ao ambiente do } \\
\text { usuário }\end{array}$ & $\begin{array}{l}\text { Adaptação dos relatórios às } \\
\text { necessidades do cliente }\end{array}$ & $\begin{array}{l}\text { Os relatórios são possíveis de serem } \\
\text { personalizados aos interesses e } \\
\text { necessidades dos clientes (como o } \\
\text { nome da fazenda, opção de combinação } \\
\text { de dados, entre outros)? }\end{array}$ & \\
\hline \multirow{5}{*}{ Conteúdo do sistema } & Correção dos resultados & $\begin{array}{l}\text { Os resultados parciais e finais são } \\
\text { corretos? }\end{array}$ & \\
\hline & Padrões de medição & $\begin{array}{l}\text { Os padrões monetários e técnicos são } \\
\text { adequados? }\end{array}$ & \\
\hline & Consistência dos dados & $\begin{array}{l}\text { Os dados e resultados parciais e finais } \\
\text { são consistentes? }\end{array}$ & \\
\hline & Rigor científico das informações & $\begin{array}{l}\text { As informações estão cientificamente } \\
\text { corretas? }\end{array}$ & \\
\hline & $\begin{array}{l}\text { Adequação das informações às } \\
\text { necessidades práticas do cliente }\end{array}$ & $\begin{array}{l}\text { O software é adequado às necessidades } \\
\text { práticas do cliente? }\end{array}$ & \\
\hline
\end{tabular}


QUADRO 2 - Características e sub-características para avaliação da qualidade de software agropecuário.

\begin{tabular}{|c|l|}
\hline ESCALA & \multicolumn{1}{|c|}{ PERCEPÇÃO } \\
\hline 0 & Muito Ruim \\
\hline 1 & Ruim \\
\hline 2 & Boa \\
\hline 3 & Muito Boa \\
\hline 4 & Ótima \\
\hline
\end{tabular}

\section{Sub-característica - Manual do usuário}

A documentação sobre o uso do software é de fácil compreensão pelo usuário?

Não, nota 1. Não possuia manual do usuário impresso. A empresa informou que o manual é on-line, porém este era muito pobre do ponto de vista técnico de informações referentes à pecuária. A tecla $\mathrm{F} 1$ oferecia ajuda on-line sensível ao contexto, porém com informações muito superficiais para ajudarem nos esclarecimentos do usuário. Possuia um roteiro explicativo de como começar a trabalhar com o sistema que foi muito útil nas simulações, também disponível apenas on-line. Mesmo sendo usado esporadicamente, o manual do usuário impresso é um recurso poderoso no esclarecimento de dúvidas quando bem elaborado e atualizado, o que não ocorreu com este sistema.

\section{CARACTERÍSTICA-FACILIDADEDE OPERAÇÃO}

\section{Sub-característica - Simplicidade para registrar informações}

É simples a entrada de dados de natureza física, zootécnica, financeira e econômica no software?

Sim, nota 4. Possuía menu intuitivo para o preenchimento de dados, ou seja, na tela de lançamentos apresentava comandos representados por botões grandes que, além de possuírem figuras sugestivas para sua operação, apresentavam o nome da rotina. Como exemplo, cita-se um comando para cadastramento de ficha individual que apresenta a figura de uma vaca e embaixo escrito "ficha". Além disso, possuía uma caderneta de campo virtual que agilizava o registro de rotinas individuais como pesagem, controle leiteiro, manejos sanitários, partos, mudança de categoria e outros, sem precisar entrar na ficha do animal.
Sub-característica - Facilidade de compreensão dos resultados

É fácil ao usuário compreender os resultados parciais e finais fornecidos pelo software?

Sim, nota 3. Os relatórios de saída de dados eram, em sua maioria, muito claros embora o usuário ficasse limitado aos modelos de relatórios pré-existentes no sistema.

\section{Sub-característica - Facilidade de consulta dos dados}

É fácil consultar os dados armazenados?

Sim, nota 4. A interface era bem clara e possuia várias rotinas de filtros para seleção de critérios com os períodos a serem consultados. Na parte zootécnica, existiam diversos filtros que possibilitaram a seleção de vários dados e, a partir daí, imprimiram-se os relatórios mais adequados.

\section{Sub característica - Facilidade de alteração dos dados}

É fácil alterar os dados?

Sim, nota 4. Existia um comando especial para alteração em várias rotinas. Quando ele não estava presente, bastava clicar duas vezes no lançamento, realizar a mudança do dado e depois gravá-lo.

\section{CARACTERÍSTICA - SERVIÇOS OFERECIDOS PELO VENDEDOR}

\section{Sub-característica - Direito a upgrade}

A aquisição do software garante o direito a upgrade gratuito ou a preço compatível?

Sim, nota 1. A empresa garantia upgrade, porém o preço para quem não tem contrato de manutenção não era compatível para atualizar o sistema. Para quem tem, era "gratuito", pois com o pagamento do valor mensal o usuário recebe a atualização.

\section{Sub-característica - Suporte Técnico}

A empresa oferece suporte técnico?

Sim, nota 1 . O suporte era gratuito no primeiro mês após a aquisição, depois disso o cliente opta se vai continuar com o suporte, assinando o contrato de manutenção ou não. Se não quisesse, receberia atendimento pago por ligação que tinha valor igual ao de um contrato de manutenção, o que deixava inviável o suporte para quem não assinava tal contrato. 
QUADRO 3 - Resultados da avaliação das características e sub-características do software ALFA.

\begin{tabular}{|c|c|c|c|}
\hline CARACTERÍSTICAS & SUB-CARACTERÍSTICAS & PERGUNTAS & NOTA \\
\hline \multirow[t]{2}{*}{ Facilidade de Uso } & Facilidade de personalização & $\begin{array}{l}\text { A interface com o usuário é facilmente } \\
\text { personalizada para o uso por usuários } \\
\text { de diferentes classes e tipos? }\end{array}$ & $\begin{array}{l}\text { SIM } \\
4\end{array}$ \\
\hline & Manual do usuário & $\begin{array}{l}\text { A documentação sobre o uso do } \\
\text { software é de fácil compreensão pelo } \\
\text { usuário? }\end{array}$ & $\begin{array}{l}\mathrm{NÃO} \\
1\end{array}$ \\
\hline \multirow{4}{*}{ Facilidade de operação } & $\begin{array}{l}\text { Simplicidade para registrar } \\
\text { informações }\end{array}$ & $\begin{array}{l}\text { É simples a entrada de dados de } \\
\text { natureza física, zootécnica, financeira e } \\
\text { econômica no software? }\end{array}$ & $\begin{array}{l}\text { SIM } \\
4\end{array}$ \\
\hline & $\begin{array}{l}\text { Facilidade de compreensão dos } \\
\text { resultados }\end{array}$ & $\begin{array}{l}\text { É fácil ao usuário compreender os } \\
\text { resultados parciais e finais fornecidos } \\
\text { pelo software? }\end{array}$ & $\begin{array}{l}\text { SIM } \\
3\end{array}$ \\
\hline & Facilidade de consulta dos dados & $\begin{array}{l}\text { É fácil consultar os dados } \\
\text { armazenados? }\end{array}$ & $\begin{array}{l}\text { SIM } \\
4\end{array}$ \\
\hline & Facilidade de alteração dos dados & É fácil alterar os dados? & $\begin{array}{l}\text { SIM } \\
4\end{array}$ \\
\hline \multirow{3}{*}{$\begin{array}{l}\text { Serviços oferecidos pelo } \\
\text { vendedor }\end{array}$} & Direito a upgrade & $\begin{array}{l}\text { A aquisição do software garante o } \\
\text { direito a upgrade gratuito ou a preço } \\
\text { compatível? }\end{array}$ & $\begin{array}{l}\text { SIM } \\
1\end{array}$ \\
\hline & Suporte técnico & A empresa oferece suporte técnico? & $\begin{array}{l}\text { SIM } \\
1\end{array}$ \\
\hline & Manutenção & $\begin{array}{l}\text { A empresa oferece serviço de } \\
\text { manutenção do software? }\end{array}$ & $\begin{array}{l}\text { SIM } \\
1\end{array}$ \\
\hline Integridade do sistema & Robustez & $\begin{array}{l}\text { O software é capaz de manter o } \\
\text { processamento, a despeito da } \\
\text { ocorrência de ações inesperadas } \\
\text { (entradas de dados incorretos, } \\
\text { execuções de ações indesejadas, } \\
\text { operações de efeito grave)? }\end{array}$ & $\begin{array}{l}\text { SIM } \\
1\end{array}$ \\
\hline $\begin{array}{l}\text { Necessidade de adaptação do } \\
\text { sistema ao ambiente do } \\
\text { usuário }\end{array}$ & $\begin{array}{l}\text { Adaptação dos relatórios às } \\
\text { necessidades do cliente }\end{array}$ & $\begin{array}{l}\text { Os relatórios são possíveis de serem } \\
\text { personalizados aos interesses e } \\
\text { necessidades dos clientes (como o } \\
\text { nome da fazenda, op̧ão de combinação } \\
\text { de dados, entre outros)? }\end{array}$ & $\begin{array}{l}\text { SIM } \\
3\end{array}$ \\
\hline \multirow{5}{*}{ Conteúdo do sistema } & Correção dos resultados & $\begin{array}{l}\text { Os resultados parciais e finais são } \\
\text { corretos? }\end{array}$ & $\begin{array}{l}\text { SIM } \\
2\end{array}$ \\
\hline & Padrões de medição & $\begin{array}{l}\text { Os padrões monetários e técnicos são } \\
\text { adequados? }\end{array}$ & $\begin{array}{l}\text { SIM } \\
4\end{array}$ \\
\hline & Consistência dos dados & $\begin{array}{l}\text { Os dados e resultados parciais e finais } \\
\text { são consistentes? }\end{array}$ & $\begin{array}{l}\text { SIM } \\
2\end{array}$ \\
\hline & Rigor científico das informações & $\begin{array}{l}\text { As informações estão cientificamente } \\
\text { corretas? }\end{array}$ & $\begin{array}{l}\text { SIM } \\
3\end{array}$ \\
\hline & $\begin{array}{l}\text { Adequação das informações às } \\
\text { necessidades práticas do cliente }\end{array}$ & $\begin{array}{l}\text { O software é adequado às necessidades } \\
\text { práticas do cliente? }\end{array}$ & $\begin{array}{l}\text { SIM } \\
3\end{array}$ \\
\hline
\end{tabular}


Outros questionamentos podem ser feitos quanto a essa sub-característica e que estão relacionados à qualidade do atendimento aos usuários como: Os atendentes possuem qualificação na área? A empresa é ágil e organizada para resolver os problemas e dar retorno aos clientes? Na simulação pôde-se usar gratuitamente o suporte da empresa e ficou claro a necessidade da especialização da mão-de-obra de suporte. Percebe-se que o trabalho de suporte e manutenção é melhor desenvolvido quando existe uma equipe qualificada e exclusiva para esse trabalho.

\section{Sub-característica - Manutenção}

A empresa oferece serviço de manutenção do software?

Sim, nota 1. A partir de um valor mensal o usuário tinha direito a suporte por telefone e $e$-mail e recebia atualizações por correio ou internet. As vantagens oferecidas pela softhouse para o usuário assinar seu contrato de manutenção estavam ligadas à questão financeira, garantindo descontos em todos os demais serviços prestados pela empresa (cursos, implantação, treinamento, modificações específicas e outros).

\section{CARACTERÍSTICA - INTEGRIDADE DO SISTEMA}

\section{Sub-característica - Robustez}

O software é capaz de manter o processamento, a despeito da ocorrência de ações inesperadas (entradas de dados incorretos, execuções de ações indesejadas, operações de efeito grave)?

Sim, nota 1. Quando ocorria algum erro, o sistema apresentava na tela uma referência com informações sobre o código de erro com opções de anular, repetir ou ignorar a ação. Na maioria das vezes o erro exigiu a reinicialização do computador, não obedecendo ao comando ignorar. $\mathrm{O}$ sistema possuia uma rotina que permitia corrigir erros encontrados e também realizar uma manutenção de seus arquivos e que podia ser executada mesmo com o software fechado.

Quanto à essa sub-característica, sugere-se que o tipo de banco de dados seja revisto, pois a quantidade de erros apresentados foi grande durante o desenvolvimento da simulação.

\section{CARACTERÍSTICA - NECESSIDADE DE ADAPTAÇÃO DO SISTEMA AO AMBIENTE DO USUÁRIO}

\author{
Sub-característica - Adaptação dos relatórios às \\ necessidade do cliente
}

Os relatórios são possíveis de serem personalizados aos interesses e necessidades dos clientes (como o nome da fazenda, opção de combinação de dados, entre outros)?

Sim, nota 3. Existiam mais de cem tipos de relatórios, porém eram determinados pelo sistema. Essa formatação apresenta a vantagem de permitir ao usuário ir direto a um relatório que necessita, como por exemplo, fêmeas a vacinar contra brucelose. Entretanto, não permite a combinação de dados para um relatório que deseje especificamente, como exemplo, comparar a evolução de ganho de peso de uma cria com a evolução de ganho de peso dos pais. Nesse caso o usuário teria que tirar um relatório de cada animal para fazer a análise. Existia também a possibilidade de exportar os dados de um relatório para outros tipos de arquivos como o txt, doc, xls e lá combinar os dados que desejasse para uma futura impressão.

\section{CARACTERÍSTICA - CONTEÚDO DO SISTEMA}

\section{Sub-característica - Correção dos resultados}

Os resultados parciais e finais são corretos?

Sim, nota 2. Algumas exceções foram diagnosticadas nesta sub-característica. Embora elas não desmerecessem os resultados das outras rotinas, elas reduziram a confiança no produto. Notaram-se informações repetidas nos relatórios de transferência de animais entre pastos na mesma fazenda. Foi feita uma simulação em que a fazenda possuía dois pastos com sistema de rotação e ocorreu duplicidade do número de animais quando estes eram movimentados de um pasto para outro. Outra incorreção ocorreu na criação dos centros de custos. A estrutura do software, na parte financeira, não oferecia mecanismos automáticos de rateio, o que gerou incorreções na análise destes resultados.

Duas soluções foram usadas para contornar o problema, a primeira foi exportando para fora do sistema, com a utilização de arquivo xls (planilha eletrônica), as informações financeiras e lá elas foram manipuladas adequadamente. A segunda, foi a criação de um centro de

Ciênc. agrotec., Lavras, v. 29, n. 5, p. 1075-1082, set./out., 2005 
custos chamado "rateio", para receber as despesas comuns a mais de um centro de custo, sendo relançados os valores deste para os centros de custos que efetivamente, ao final do ano agrícola, pagaram pelas despesas. Quanto a essa sub-característica, seria necessário criar uma rotina específica para a realização de rateios na parte financeira, tão comuns nos negócios agropecuários.

\section{Sub-característica - Padrões de medição}

Os padrões monetários e técnicos são adequados?

Sim, nota 4. Os padrões monetários podiam ser indexados em quatro tipos de moedas: o dólar, o real e outros dois tipos configurados pelo usuário. Os padrões técnicos apresentavam grande flexibilidade para atender a diversos tipos de pecuária, pois os itens constantes das tabelas de parametrização do sistema, podiam ser criados pelo usuário e tanto as entradas e saídas de dados eram referentes a estes.

\section{Sub-característica - Consistência dos dados}

Os dados e resultados parciais e finais são consistentes?

Sim, nota 2. Algumas exceções foram diagnosticadas nesta sub-característica. Embora elas não desmerecessem os resultados das outras rotinas, elas reduziram a confiança no produto. Notou-se que o sistema permitia que saísse maior número de animais do que existia em estoque e este ficava negativo.

Na parte reprodutiva não previa o trabalho com a situação de repasse, que é a inseminação seguida de monta a campo. Na ficha reprodutiva das matrizes, o usuário tinha que realizar dois lançamentos de cobertura, em campos separados, o que aumentava o intervalo de partos da matriz.

\section{Sub-característica - Rigor científico das informações}

As informações estão cientificamente corretas?

Sim, nota 3. Principalmente na parte zootécnica. Poderia ter outros tipos de relatórios que explorassem os dados já existentes no sistema.

\section{Sub-característica - Adequação das informações às necessidades práticas do cliente} do cliente?

O software é adequado às necessidades práticas

Sim, nota 3. Na parte financeira há necessidade de rotinas mais sofisticadas e real integração com a parte zootécnica, pois, esta trabalhou separada da financeira gerando a necessidade do fechamento de custos da atividade fora do sistema.

Na parte zootécnica o sistema atendeu à condições tanto da pecuária de corte como da pecuária de leite e suas variantes de sistemas de produção. Na pecuária de leite, poderia oferecer maior diversidade de relatórios. A integração com balanças eletrônicas e comunicação com leitoras para código de barras foram constatadas. Os softwares que se comunicam com esses equipamentos têm ganhado a atenção dos pecuaristas, pois cada vez mais esses equipamentos têm sido procurados como uma oportunidade a mais para o atendimento das normas instituídas para o SISBOV (Sistema Brasileiro de Certificação e Identificação de Origem Bovina e Bubalina) (BRASIL, 2002).

De acordo com a avaliação feita pelos especialistas do domínio, a qualidade do software Alfa foi considerada aceitável, isto é, recomendado para uso e ou aquisição. Sugere-se, entretanto, atenção da softhouse para a melhoria da característica serviços oferecidos pelo vendedor, subcaracterísticas direito a upgrade, suporte técnico e manutenção, pois foi identificado suporte não adequado e condições de comercialização das atualizações desfavoráveis.

\section{CONSIDERAÇÕES FINAIS}

Não existe um modelo ideal de avaliação de qualidade que seja aplicável indistintamente aos softwares agropecuários, pois, a qualidade depende de opiniões subjetivas de quem está analisando, passando a ser mais precisa à medida que o usuário conhece e utiliza o software. Isso significa dizer que, se a avaliação do software Alfa fosse submetida a outros especialistas de domínio, sua qualidade poderia não ser considerada aceitável. Entretanto, cabe ressaltar a importância de uma avaliação técnica, já que a qualidade é um fator diferenciador dos produtos disponíveis comercialmente.

A forma de comercialização da softhouse vem mudando, pois, o software não é mais comercializado apenas como um produto, mas sim como um pacote de serviços, no qual o suporte pós-venda ao usuário é fundamental para a sobrevivência da empresa e isto exige a identificação de novos atributos, que caracterizem melhor a proposta e as necessidades dos usuários.

A maior preocupação da softhouse deve ser sempre a satisfação do usuário final, pois é ele quem define as características de qualidade de um produto de software. 
Poucas são as empresas de software que consideram a qualidade como um fator competitivo e o mercado vem demonstrando que, no setor agropecuário, a qualidade é fator decisivo na hora da aquisição do produto.

Os usuários que estejam interessados em avaliar a qualidade de produtos agropecuários, sejam eles produtores rurais, zootecnistas, engenheiros agrônomos ou desenvolvedores de software, podem utilizar esta proposta como ponto de partida para a definição de um modelo de avaliação dos produtos de software vendidos comercialmente.

\section{REFERÊNCIAS BIBLIOGRÁFICAS}

BOAVENTURA, I. A. G. Qualidade de Software. [S.l.: s.n.], 2001. Notas de aula, Engenharia de Software.

BRASIL. Instrução Normativa n.1, 9 jan. 2002. Institui o Sistema Brasileiro de Identificação e Certificação de Origem Bovina e Bubalina - SISBOV. [S.1.: s.n.], 2002.

CAMPOS, F.; BRAGA, R.; COELHO, F. Quality process to improve agricultural software products. Germany: FEESMA, 2000.
CAMPOS, F. C. A. Qualidade de software agropecuário. In: FEIRA E CONGRESSO DE INFORMÁTICA APLICADA À AGROPECUÁRIA E AGROINDÚSTRIA, 1999, Juiz de Fora. Anais... Juiz de Fora: Softex, 2000. CDROM.

CAMPOS, F. C. A. Avaliação de software agropecuário. In: ROCHA, A. R. C.; MALDONADO, J. C.; WEBER, K. C. Qualidade de software: teoria e prática. São Paulo: Prentice Hall, 2001. p. 208-216.

CÓCARO, H. Avaliação do uso de softwares para gerenciamento de rebanhos bovinos leiteiros no sul de Minas Gerais: um estudo de caso. 2004. 100 f. Monografia (Especialização em Administração Rural) - Universidade Federal de Lavras, Lavras, 2004.

ISO/IEC 9126-1. Software engineering: product quality. [S.1.], 2001.

LOPES, M. A. Sistema computacional para dimensionamento de rebanhos bovinos utilizando valores ajustados de equivalência das categorias animais. 2000. 116 f. Tese (Doutorado em Zootecnia) - Universidade Estadual Paulista, Jaboticabal, 2000. 